\title{
Zum Einfluss kurzfristiger Fremdfinanzierungsmöglichkeiten auf die Portfoliooptimierung mit illiquiden Assets
}

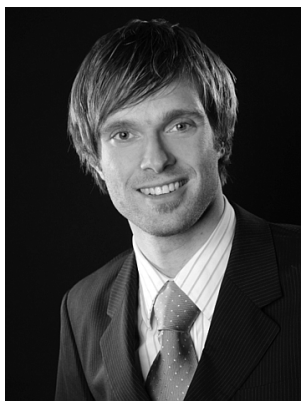

\section{Dennis Diepold}

Portfoliooptimierung, Privatanleger, Kredit, Fremdfinanzierung, Illiquidität, illiquide Assets

Portfolio optimization, private investor, credit, borrowing, illiquidity, illiquid assets

Bei der Überlegung, als Privatanleger in ein illiquides Asset wie bspw. eine Immobilie zu investieren, spielt ein (möglicher) künftiger Liquiditätsbedarf eine gewichtige Rolle. Geht man davon aus, dass illiquide Assets nur vollständig und mit Wertabschlag auch kurzfristig verkauft werden können, so kann ein Liquiditätsbedarf dazu führen, dass das illiquide Asset kurzfristig liquidiert oder ein Kredit aufgenommen werden muss, wodurch jeweils Kosten entstehen. In der heutigen Anlageberatung wird dagegen meist ein pauschales Liquiditätspolster empfohlen und das Portfolio des Kunden unabhängig davon oder von illiquiden Assets optimiert. Der vorliegende Beitrag analysiert die Auswirkungen einer kurzfristigen Fremdfinanzierungsmöglichkeit auf die Portfoliooptimierung mit illiquiden Assets. Er zeigt auf, dass diese die Nachteile eines illiquiden Assets teilweise reduziert und daher - wie auch die Besonderheiten illiquider Assets selbst - unbedingt bei der Portfoliooptimierung berücksichtigt werden muss.

When considering an investment into illiquid assets such as real estate, (possible) future liquidity requirements play an essential role for private investors. We assume that illiquid assets can only be sold as a whole, and, on a short term basis, only with a discount. Because of a liquidity requirement the illiquid asset may have to be sold or the investor may have to borrow money, with both alternatives leading to costs. Nevertheless, today's investment consulting often recommends a lump-sum liquidity reserve, optimizing the portfolio independent of this reserve or of illiquid assets. This paper analyzes the impact of short-term borrowing on portfolio optimization with illiquid assets. It shows that shortterm borrowing reduces the disadvantages of illiquid assets and thus, same as the constituting properties of illiquid assets, has to be considered within any portfolio optimization.

\section{Einleitung}

Spätestens seit der Finanz- und Wirtschaftskrise hat die Problemstellung, die sich aus der Illiquidität von Assets ergibt, wieder an Aktualität und Aufmerksamkeit in Forschung und Praxis gewonnen. Obwohl bekannt ist, dass Illiquidität erheblichen Einfluss auf die Ergebnisse einer Portfoliooptimierung hat (bspw. Grossman/Laroque 1990; Faig/Shum 2002; Huang 2003; Longstaff 2009), wurde dieser Einfluss sowohl bei Kreditinstituten als auch Privatanlegern unterschätzt. 
Heutige Anlageberatung geht auf diese Problematik in der Regel nur sehr oberflächlich ein (Diepold 2011). Meist wird dem Anleger dazu geraten, zunächst einen gewissen Anteil des Vermögens als Liquiditätsreserve vorzuhalten. Mit dem verbleibenden Kapital wird ein Portfolio angestrebt, welches die Präferenzen des Kunden möglichst gut abbildet und dabei möglichst nah an einer Rendite-/Risiko-effizienten Lösung liegt. Illiquides Vermögen wird meist unabhängig davon betrachtet oder ganz vernachlässigt (Rudolf 2008). Elton/Gruber (2000) zeigen beispielsweise von grossen Investment Banken vorgeschlagene Asset-Allokationen, welche zumeist pauschal einen Bargeldanteil von 5\%, einige Male sogar 50\% vorgeben und den Rest auf andere Assets (Anleihen und Aktien) verteilen. Offensichtlich wird die Illiquidität der Assets damit nicht hinreichend berücksichtigt und Optimierungspotenzial verschenkt.

Einer der möglichen Gründe dafür, dass die bisherigen Modelle zur Berücksichtigung von Illiquidität bei der Portfoliooptimierung keine Anwendung in der privaten Anlageberatung finden, mag darin liegen, dass diese die Möglichkeit einer Kreditaufnahme ausschliessen. Damit wird insbesondere vernachlässigt, dass der Anleger im Falle eines Liquiditätsbedarfs, welcher grösser als die zur Verfügung stehende Liquidität ist, nicht zwingend auf seine illiquiden Assets zugreifen muss, sondern die benötigte Liquidität oftmals auch über einen Kredit kurzfristig beschaffen kann. Besitzt der Anleger bspw. eine Liquiditätsanforderung, die nur geringfügig grösser als seine Liquiditätsreserve ist, so wird er in der Regel nicht seine Immobilie verkaufen, sondern den Fehlbetrag fremdfinanzieren. Der vorliegende Beitrag widmet sich dieser Forschungslücke und untersucht den Einfluss einer solchen Fremdfinanzierungsmöglichkeit bei eintretender Liquiditätsanforderung auf die optimale Anlageentscheidung mit liquiden und illiquiden Assets. Dabei wird bspw. gezeigt, dass die kurzfristige Fremdfinanzierungsmöglichkeit die Nachteile der Illiquidität derart verringert, dass eine höhere Portfoliorendite bei gleichzeitig geringerem Risiko ermöglicht wird.

Der Beitrag gliedert sich wie folgt: Im folgenden Abschnitt 2 wird Asset-Liquidität definiert und ihre Relevanz dargelegt. Aus einem Überblick über die relevante Literatur zur Portfoliooptimierung mit illiquiden Assets werden anschliessend die Forschungslücke sowie die konkreten Forschungsfragen abgeleitet. In Abschnitt 3 wird ein quantitatives Modell zu deren Beantwortung vorgestellt. Dieses wird in Abschnitt 4 analysiert und Implikationen einer Fremdfinanzierungsmöglichkeit für die Portfoliooptimierung mit illiquiden Assets herausgearbeitet. In Abschnitt 5 werden die Ergebnisse kritisch diskutiert und Abschnitt 6 fasst die Inhalte des Beitrags abschliessend zusammen.

\section{Literaturüberblick \& Forschungsfragen}

\subsection{Definition und Relevanz von Asset-Liquidität}

Schon Anfang der 30er Jahre definierte Keynes (1932, 348 f.) liquide Assets „in dem Sinne, dass sie mit grösserer Sicherheit in kurzer Frist ohne Verlust realisiert werden können“. Damit wies er bereits auf die beiden entscheidenden Parameter hin, welche bei der Definition von Asset-Liquidität eine entscheidende Rolle spielen:

(1) Der Zeitraum, der für den Verkauf des Assets benötigt wird, und

(2) der Preis, der dadurch erzielt wird.

Ein perfekt liquides Asset ist demnach jederzeit und ohne Preisabschlag handelbar (Kempf 1998). Daraus folgt, dass ein illiquides Asset (wenn überhaupt) nur zeitverzögert gehan- 
delt werden kann (Lippman/McCall 1986; Longstaff 2009) oder dessen kurzfristiger Verkauf zwar möglich, aber nur mit entsprechendem Preisabschlag realisierbar ist (Grossman/ Laroque 1990; Faig/Shum 2002; Ericsson/Renault 2006). Ein jederzeit möglicher Handel illiquider Assets wird beispielsweise mit der Existenz von sog. „market makers“ begründet, welche zur Erhaltung des Markts illiquide Assets zwar unmittelbar, jedoch gegen einen entsprechenden Preisabschlag kaufen (Grossman/Miller 1987; Heflin/Shaw 2000). Zudem erscheint es durchaus denkbar, dass man beispielsweise eine Immobilie ebenfalls sehr kurzfristig verkaufen kann, wenn man bereit ist, einen deutlich niedrigeren Preis als den aktuellen Marktwert zu akzeptieren (Grossman/Laroque 1990; Diepold/Dzienziol 2009). Aufbauend auf der Arbeit von Diepold/Dzienziol (2009) wird die dort verwendete Definition illiquider Assets (kurzfristiger Verkauf mit Preisabschlag möglich) auch im vorliegenden Beitrag zu Grunde gelegt.

Da der Grad der Illiquidität im vorliegenden Beitrag vorgegeben ist, steht deren Messung nicht im Fokus. Einen Überblick über verschiedene Konzepte zur Liquiditätsmessung liefern bspw. Bernstein (1987), Kempf (1998), Sibilkov (2009) oder Jankowitsch et al. (2011).

Gemäss ihrer Definition wird Illiquidität dann relevant, wenn Assets kurzfristig gehandelt werden müssen. Dies ist beispielsweise der Fall, wenn (zu bestimmten oder zufälligen Zeiten) Liquiditätsbedarfe bestehen, die nicht aus dem liquiden Vermögen gedeckt werden können (Diepold/Dzienziol 2009), oder eine Asset-Reallokation notwendig ist (Ericsson/ Renault 2006). Ein weiterer Grund für die Notwendigkeit einer kurzfristigen Liquidation liegt darin, dass in der Regel Konsum ausschliesslich über liquide Mittel eigenfinanziert werden kann und Konsumpräferenzen nicht zeitkonstant sind (Diamond/Dybvig 1983; Huang 2003).

\subsection{Illiquide Assets in der Portfoliooptimierung}

Die in der bisherigen Literatur präsentierten quantitativen Modelle zur Berücksichtigung illiquider Assets bei der Portfoliooptimierung besitzen aufgrund der Komplexität der Fragestellung jeweils sehr spezifische Rahmenbedingungen und analysieren oft eine Entscheidung zwischen zwei Assets. Sie lassen sich dabei gemäss obiger Definition von Liquidität wie folgt klassifizieren: Illiquide Assets können entweder (1) gar nicht, (2) nicht jederzeit oder (3) jederzeit unter Transaktionskosten gehandelt werden.

\subsubsection{Nicht handelbare Assets}

Bei der Betrachtung nicht handelbarer Assets wird in der Regel davon ausgegangen, dass diese zu einer (regelmässigen) liquiden Rendite führen. Ein besonders prominentes Beispiel für diese Art eines illiquiden Assets ist Humankapital (Spremann/Winhart 1997; Schwartz/ Tebaldi 2006), bei welchem bspw. durch den Einsatz von Arbeitskraft Einzahlungen generiert werden. Arbeiten auf diesem Gebiet liegt daher in der Regel ein vorgegebenes illiquides Asset mit fixem Anteil zu Grunde, welches nicht gehandelt werden kann und zu Einzahlungen führt. Gegenstand der Untersuchung ist dann die Asset-Allokation auf unterschiedlich riskante liquide Assets, abhängig von der jeweiligen Korrelation mit dem illiquiden Asset, dessen Anteil oder Rendite. Der optimale Anteil des illiquiden Assets wird folglich nicht untersucht.

Einer ähnlichen Fragestellung widmen sich Faig/Shum (2002), wobei diese statt Humankapital ein sog. illiquides Projekt (wie bspw. einen Hausbau) betrachten. Dieses kann aus- 
gehend von einem in der ersten Periode gewählten Startwert in der zweiten Periode entweder mit einem proportional festgelegten Betrag weiterfinanziert oder vollständig abgebrochen werden. Ein Teil des benötigten Kapitals kann dabei auch geliehen werden. Die Autoren kommen zu dem Ergebnis, dass Anleger weniger riskante Assets in ihr Portfolio aufnehmen, je höher der Nutzen aus dem illiquiden Projekt und der Verlust durch dessen Abbruch sind.

\subsubsection{Nicht jederzeit handelbare Assets}

Vollständige Relevanz im Rahmen einer ganzheitlichen Asset-Allokation erhält Asset-Illiquidität allerdings erst dann, wenn auch illiquide Assets handelbar sind und deren Anteil ebenfalls optimiert wird. In den folgenden Arbeiten (Koren/Szeidl 2002; Longstaff 2009; Gârleanu 2009) wird angenommen, dass dieser Handel nicht jederzeit möglich ist.

Koren/Szeidl (2002) betrachten die Entscheidung zwischen einem liquiden und einem illiquiden Asset - beide mit sicheren Renditen - wobei das illiquide Asset nur zeitverzögert gehandelt werden kann, dafür jedoch eine höhere Rendite als das liquide Asset besitzt. Der Anleger kann Konsum tätigen, dessen Nutzen während zufällig auftretender sog. „liquidity shocks" erhöht ist und der ausschliesslich über die vorhandene Liquidität finanziert werden kann. Koren/Szeidl (2002) stellen u. a. fest, dass Anleger mit langem Horizont überwiegend in illiquide Assets investieren, während sie bei kurzem Horizont eher Liquidität präferieren.

Longstaff (2009) analysiert in einer Tauschwirtschaft mit zwei Marktteilnehmern deren Analageentscheidung zwischen einem liquiden und einem illiquiden riskanten Asset. Das illiquide Asset kann über einen definierten Zeitraum (sog. „,blackout' period“) nicht gehandelt werden. Auch hier wird aus dem Portfolio Konsum finanziert. Longstaff (2009) kommt u. a. zu dem Ergebnis, dass die Anleger aufgrund der Illiquidität nicht mehr das Marktportfolio wählen, sondern eher zu stark polarisierten Portfolios neigen. Passend zu den Ergebnissen von Koren/Szeidl (2002) investieren „ungeduldige“ Anleger (die früher konsumieren wollen) stärker in das liquide, „geduldige“ Anleger stärker in das illiquide Asset.

Gârleanu (2009) legt seiner Analyse ein ähnliches Setting zugrunde. Bei der Bestimmung des optimalen Portfolios aus einem risikolosen und einem riskanten Asset nimmt er allerdings an, dass alle Assets nur zu bestimmten Zeitpunkten gehandelt werden können. Zentraler Untersuchungsgegenstand ist dabei der Einfluss der Marktliquidität auf die Portfoliostruktur eines risikoaversen Anlegers. Im Gegensatz zu Longstaff (2009) kommt er zu dem Ergebnis, dass Anleger eher extreme Positionen eingehen, wenn diese einen sehr liquiden Markt erwarten, da in diesem Fall leichter gehandelt werden kann.

\subsubsection{Jederzeit mit Preisabschlag handelbare Assets}

Den folgenden Arbeiten von Grossman/Laroque (1990), Flavin/Yamashita (2002), Huang (2003) sowie Diepold/Dzienziol (2009) liegt schliesslich die Annahme zu Grunde, dass auch illiquide Assets jederzeit handelbar sind, wenn man einen entsprechend geringen Preis dafür ansetzt.

Grossman/Laroque (1990) untersuchen folgende Situation: Eine Privatperson besitzt als illiquides Asset ein sog. „durable consumption good“ (bspw. ein Haus) mit stetig abnehmendem Wert, welches nur vollständig gehandelt werden kann, und dessen Verkauf mit 
Preisabschlag kurzfristig möglich ist. Der Anleger hat die Möglichkeit in ein sicheres und mehrere unsichere liquide Assets zu investieren, um sein Vermögen zu steigern und langfristigen Konsum zu sichern. Sein Konsumlevel ist dabei proportional zum Wert des illiquiden Assets und kann durch Kauf eines Neuen (bei Verkauf des Alten) gesteigert werden. Grossman/Laroque (1990) kommen zu dem Ergebnis, dass es einen Bereich des aktuellen Konsumlevels gibt, in welchem das dauerhafte Gut unangetastet bleibt.

Auf Basis des Beitrags von Grossman/Laroque (1990) untersuchen Flavin/Yamashita (2002) einen Anleger mit einer selbstgenutzten Immobilie als spezielles illiquides Asset. Der Anleger kann bereits bei seiner initialen Anlageentscheidung einen Teil der Immobilie fremdfinanzieren, d. h. einen Leverage im Portfolio erzeugen. Mögliche Liquiditätsanforderungen bleiben jedoch weiterhin unberücksichtigt. Ergebnis des Beitrags ist, dass junge Haushalte mit hohem Leverage bereits durch diesen eine hohe Risikoposition besitzen und daher ihr restliches Portfolio eher risikoarm gestalten, wohingegen ältere Haushalte mit niedrigem Leverage riskantere liquide Assets im Portfolio haben.

Huang (2003) untersucht in einem Modell mit jeweils einem risikolosen liquiden und illiquiden Bond mit gleichem Zins den Einfluss zufällig auftretender Liquiditätsanforderungen auf die optimale Anlagestrategie und die Liquiditätsprämie. Er unterscheidet dabei zwei Fälle:

1. Der Anleger erhält ein regelmässiges Einkommen zur Erfüllung der Liquiditätsanforderungen.

2. Dem Anleger steht ausschliesslich ein initiales Vermögen zur Verfügung. Damit nimmt Huang ebenfalls eine langfristige Fremdfinanzierungsmöglichkeit an, worüber das künftige Einkommen initial zur Verfügung gestellt werden kann. Darüber hinausgehende Fremdfinanzierungen zur kurzfristigen Überbrückung von Liquiditätsbedarfen sind jedoch ausgeschlossen. Des Weiteren bleiben die Kosten, die durch die frühzeitige Bereitstellung des Vermögens entstehen, und damit der eigentliche Trade-off zwischen Liquidation und Kreditaufnahme unberücksichtigt.

Huang (2003) kommt zu dem Ergebnis, dass Illiquidität - repräsentiert durch Transaktionskosten bei Verkauf - im 1. Fall starke Effekte auf die Renditen von Assets hat.

Auch Diepold/Dzienziol (2009) nehmen an, dass illiquide Assets nur vollständig verkauft werden können und ein kurzfristiger Verkauf zu Verlusten führt. Im Rahmen einer Portfoliooptimierung mit einem liquiden und einem illiquiden Asset stellen die Autoren bspw. fest, dass die angenommene zufällige Liquiditätsanforderung zur Folge hat, dass der Portfoliowert auch bei sicheren Renditen der Assets bereits unsicher ist. Damit kann trotz der sicheren Renditen eine Aufteilung sowohl auf liquide als auch illiquide Assets optimal sein. Nach sukzessivem Hinzufügen von Unsicherheit bzgl. der jeweiligen Renditen wird des Weiteren gezeigt, dass der erwartete Portfoliowert abhängig von der Varianz der Assets sein kann und sich eine negative Korrelation zwischen Assets ggf. nachteilig auf diesen auswirkt.

\subsection{Forschungsfragen}

Im vorliegenden Beitrag wird unter einer kurzfristigen Fremdfinanzierung ausschliesslich die Überbrückung kurzfristiger Liquiditätsengpässe durch Fremdkapital verstanden. Eine kurzfristige Fremdfinanzierung erfolgt demnach nur dann, wenn das bestehende Portfolio nicht genügend Liquidität beinhaltet, um eine akute Liquiditätsanforderung zu decken (bspw. über einen Konsumentenkredit). 
Im Gegensatz dazu steht die langfristige Fremdfinanzierung, bei welcher das Fremdkapital zur Finanzierung des Portfolios selbst verwendet wird (bspw. Immobilienkredit). Diese wird im Folgenden nicht berücksichtigt. Erste Ergebnisse in diesem Bereich liefert der bereits vorgestellte Beitrag von Flavin/Yamashita (2002).

Wie Tabelle 1 nochmals zusammenfassend aufzeigt, berücksichtigen nur wenige der dargestellten Ansätze überhaupt mögliche Liquiditätsanforderungen. Dabei beinhaltet keiner dieser Aufsätze die Möglichkeit, eine entsprechende Liquiditätsanforderung auch kurzfristig fremdfinanzieren zu können. Damit werden die bisherigen Ansätze den in der Regel vorherrschenden Rahmenbedingungen nicht gerecht.

\begin{tabular}{|l|c|c|l|}
\hline Beitrag & Handelbarkeit & Liquiditätsanforderung & $\begin{array}{l}\text { Kurzfristige } \\
\text { Fremdfinanzierung }\end{array}$ \\
\hline Faig/Shum (2002) & keine & \multicolumn{2}{|c|}{ nicht berücksichtigt } \\
\hline Koren/Szeidl (2002) & teilweise & berücksichtigt & nicht berücksichtigt \\
\hline Longstaff (2009) & teilweise & \multicolumn{2}{|c|}{ nicht berücksichtigt } \\
\hline Gârleanu (2009) & teilweise & \multicolumn{2}{|c|}{ nicht berücksichtigt } \\
\hline Grossman/Laroque (1990) & Preisabschlag & \multicolumn{2}{|c|}{ nicht berücksichtigt } \\
\hline Flavin/Yamashita (2002) & Preisabschlag & \multicolumn{2}{|c|}{ nicht berücksichtigt } \\
\hline Huang (2003) & Preisabschlag & berücksichtigt & nicht berücksichtigt \\
\hline Diepold/Dzienziol (2009) & Preisabschlag & berücksichtigt & nicht berücksichtigt \\
\hline
\end{tabular}

Tabelle 1: Relevante Literatur

Der vorliegende Aufsatz widmet sich explizit dieser Forschungslücke und liefert einen Beitrag zur Beantwortung der folgenden Forschungsfragen:

Welchen Einfluss hat eine kurzfristige Fremdfinanzierungsmöglichkeit auf die optimale Anlageentscheidung mit liquiden und illiquiden Assets und wovon ist diese abhängig?

Welche Alternative zur Bereitstellung ausreichender Liquidität im Falle einer Liquiditätsanforderung (kurzfristige Liquidation des illiquiden Assets oder Fremdfinanzierung) ist wann vorteilhaft?

\section{Modell zur Berücksichtigung von Illiquidität und der Möglichkeit kurzfristiger Fremdfinanzierung eines Liquiditätsbedarfs}

Als Grundlage zur Beantwortung dieser Fragen bietet sich aufgrund des bereits dargelegten Verständnisses von Asset-Liquidität sowie der expliziten Berücksichtigung einer Liquiditätsanforderung insbesondere das Modell von Diepold/Dzienziol (2009) an. Dieses wird im Folgenden derart erweitert, dass die Möglichkeit kurzfristiger Fremdfinanzierung Berücksichtigung findet.

(A1) Zielfunktion: Der Anleger will einen derzeit verfügbaren Betrag für zwei Perioden gleicher Länge anlegen. Dabei stehen ihm eine liquide und eine illiquide Assetklasse mit den prozentualen Anteilen $x_{1} \in[0 ; 1]$ und $1-x_{1}$ am Portfolio $x=\left(x_{1}, 1-x_{1}\right)^{T}$ zur Verfügung. Durch die einmalige Festlegung des Anteils des liquiden Assets am Ge- 
samtportfolio zu Beginn der ersten Periode soll der erwartete Portfoliowert am Ende der zweiten Periode $E\left(V\left(x_{1}\right)\right)$ maximiert werden.

Der Anlagebetrag wird im Folgenden ohne Beschränkung der Allgemeinheit auf 1 normiert.

(A2) Nebenbedingung: Die Risikoaversion des Anlegers wird dadurch abgebildet, dass der Portfoliowert am Ende der zweiten Periode in jedem Fall einen Mindestwert in Höhe von $\omega>0$ erreichen soll.

Die getrennte Modellierung von Rendite und Risiko erlaubt es, die betrachteten Einflüsse und Auswirkungen differenziert zu analysieren. ${ }^{1}$

Wie bereits beschrieben, kommt den Besonderheiten illiquider Assets insbesondere dann eine Bedeutung in der Portfoliooptimierung zu, wenn die Notwendigkeit zur Entnahme liquider Mittel aus dem Portfolio bestehen könnte.

(A3) Liquiditätsanforderung: Mit Wahrscheinlichkeit $p_{l}>0$ benötigt der Anleger am Ende der ersten Periode einen Betrag $I>0$ zur Entnahme. Zur Deckung dieses Liquiditätsbedarfs kann es nötig sein, die Portfoliostruktur am Ende der ersten Periode anzupassen.

Die zur Verfügung stehenden Anlagemöglichkeiten werden wie folgt charakterisiert:

(A4) Assets: Die Wertentwicklungen des liquiden $\left(\mu_{1}\right)$ und des illiquiden Assets $\left(\mu_{2}\right)$ sind sicher und jeweils für beide Perioden gleich. ${ }^{2}$ Das illiquide Asset kann nur vollständig gehandelt werden. Beide Assets sind kurzfristig liquidierbar, wobei die kurzfristige Liquidation des illiquiden Assets mit prozentualen Kosten $c \in(0 ; 1]$ verbunden ist. Am Ende der zweiten Periode kann das illiquide Asset ohne Wertverlust liquidiert werden. Ein durch den Verkauf des illiquiden Assets erzielter, über den Liquiditätsbedarf hinausgehender Betrag wird unmittelbar in das liquide Asset reinvestiert.

Die Auswirkungen der Illiquidität werden demnach direkt monetarisiert. Andere Kaufoder Verkaufstransaktionskosten von Assets werden nicht betrachtet (vgl. bspw. Grossman/Laroque 1990; Huang 2003). Die Länge der zweiten Periode entspricht beispielsweise der Restlaufzeit einer Lebensversicherung oder der benötigten Zeit, bis eine Immobilie zum geplanten Verkehrswert verkauft werden kann. Nochmals hervorzuheben ist, dass zu jedem beliebigen Anlagebetrag ein passendes illiquides Asset erworben werden kann (vgl. bspw. Grossman/Laroque 1990).

Durch die sichere Wertentwicklung kennt der Anleger den Portfoliowert zum Zeitpunkt der Liquiditätsanforderung. Er konsumiert nicht mehr als sein verfügbares Kapital:

(A5) Höhe der Liquiditätsanforderung: Die Liquiditätsanforderung des Anlegers ist nicht grösser als der Portfoliowert am Ende der ersten Periode bei vollständiger Liquidation $\left(I \leq x_{1} \mu_{1}+\left(1-x_{1}\right) \mu_{2}(1-c)\right)$.

Neben der kurzfristigen Liquidation des illiquiden Assets besitzt der Anleger zudem die Möglichkeit, die am Ende der ersten Periode eventuell benötigte und über den Wert des

1 Es handelt sich hierbei um einen Spezialfall des Telser-Kriteriums (Telser 1955), welches in Diepold/ Dzienziol (2009) ausführlich motiviert wird.

2 Im Einklang mit der in Abschnitt 2.2 dargestellten Literatur, insbesondere dem Modell von Diepold/ Dzienziol (2009), werden Steuern nicht berücksichtigt. Im Gegensatz zu Diepold/Dzienziol (2009) beschränkt sich das hier vorgestellte Modell auf Grund der erhöhten Komplexität mit zwei Perioden und zwei Finanzierungsalternativen zudem auf Assets mit sicheren Renditen. Dies ermöglicht die nachvollziehbare Herleitung erster konkreter Ergebnisse und entspricht einem gängigen Vorgehen (vgl. bspw. Koren/Szeidl 2002; Huang 2003). 
liquiden Assets hinausgehende Liquidität durch Aufnahme eines Kredits bis zur verlustfrei möglichen Veräusserung des illiquiden Assets kurzfristig fremd zu finanzieren.

(A6) Kredit: Als dritte Assetklasse, mit nicht-positivem Anteil $x_{3} \leq 0$ des Anlagebetrags, steht am Ende der ersten Periode ein unbegrenzter Kredit mit sicherer Wertentwicklung $\mu_{3}(1+$ Effektivzins $)$ zur Verfügung. Der Effektivzins des Kredits ist dabei stets grösser gleich der Renditen der beiden anderen Assets $\left(\mu_{3} \geq \max \left\{\mu_{1} ; \mu_{2}\right\}\right){ }^{3}$ Zins- und Tilgungszahlungen erfolgen vollständig am Ende der Laufzeit (d. h. am Ende der zweiten Periode).

Zur Untersuchung der genannten Forschungsfragen werden im Folgenden zunächst die möglichen Ereignisse zu den einzelnen Zeitpunkten verdeutlicht und im Anschluss daran Zielfunktion und Nebenbedingung getrennt voneinander analysiert.

\section{Analyse des Modells}

$\mathrm{Zu}$ Beginn der ersten Periode investiert der Anleger die Anteile $x_{1}$ und $\left(1-x_{1}\right)$ in das liquide und illiquide Asset, welche somit am Ende der ersten Periode den Wert $x_{1} \mu_{1}$ und $\left(1-x_{1}\right) \mu_{2}$ besitzen.

Am Ende der ersten Periode können folgende Situationen auftreten:

(1) Es tritt kein Liquiditätsbedarf ein (Wahrscheinlichkeit $\left(1-p_{l}\right)$ ).

(2) Es tritt ein Liquiditätsbedarf ein (Wahrscheinlichkeit $p_{I}$ ), welcher entweder...

a. ... vollständig durch den Wert des liquiden Assets gedeckt werden kann, oder

b. ... grösser als der Wert des liquiden Assets ist.

Situation (1): Das Portfolio bleibt bis zum Ende der zweiten Periode unverändert und es folgt der Portfoliowert $V_{(1)}\left(x_{1}\right)=x_{1} \mu_{1}^{2}+\left(1-x_{1}\right) \mu_{2}^{2}$.

Situation (2 a): Der Liquiditätsbedarf $l$ wird vollständig aus dem liquiden Asset entnommen und man erhält den Portfoliowert $V_{(2 a)}\left(x_{1}\right)=\left(x_{1} \mu_{1}-I\right) \mu_{1}+\left(1-x_{1}\right) \mu_{2}^{2} \cdot 4$

Situation (2 b): Nach vollständiger Entnahme des liquiden Assets hat der Anleger folgende Möglichkeiten, um die restliche Liquidität $\left(I-x_{1} \mu_{1}\right)$ bereitzustellen:

(L) Verkauf des illiquiden Assets (welches den Wert $\left(1-x_{1}\right) \mu_{2}(1-c)$ erzielt) und Reinvestition des eventuell über den Bedarf hinausgehenden Betrags

$\left(x_{1} \mu_{1}+\left(1-x_{1}\right) \mu_{2}(1-c)-I\right)$ in das liquide Asset. Damit ergibt sich der Portfoliowert $V_{(2 b), L}\left(x_{1}\right)=\left(x_{1} \mu_{1}+\left(1-x_{1}\right) \mu_{2}(1-c)-I\right) \mu_{1}$.

(K) Aufnahme des Kredits in Höhe des noch verbleibenden Betrags. Man erhält den Portfoliowert $V_{(2 b), K}\left(x_{1}\right)=-\left(I-x_{1} \mu_{1}\right) \mu_{3}+\left(1-x_{1}\right) \mu_{2}^{2}$.

Tabelle 2 zeigt die Auswirkungen der einzelnen Fälle auf die Werte der Assets.

3 Der Effektivzins entspricht den tatsächlich entstehenden prozentualen Kosten des Anlegers für die Geldaufnahme (d. h. dem Nominalzins plus evtl. Disagio, Gebühren etc.).

4 Den Liquiditätsbedarf bereits in dieser Situation durch Liquidation des illiquiden Assets zu tilgen, wäre aufgrund der Wiederanlage im liquiden Asset nur dann sinnvoll, wenn die Rendite des illiquiden Assets in der zweiten Periode kleiner als die des liquiden Assets abzüglich der Liquidationskosten ist $\left(\mu_{2}<(1-c) \mu_{1}\right)$. In diesem Fall wird allerdings vollständig in das liquide Asset investiert. 


\begin{tabular}{|l|c|c|c|}
\hline Situation & Liquides Asset & Illiquides Asset & Kredit $(<0)$ \\
\hline \multicolumn{3}{|c|}{ Ende der 1 Periode } \\
\hline $1-2 \mathrm{~b}$ & $x_{1} \mu_{1}$ & $\left(1-x_{1}\right) \mu_{2}$ & 0 \\
\hline \multicolumn{4}{|c|}{ Ende der 2 . Periode } \\
\hline 1 & $x_{1} \mu_{1}^{2}$ & $\left(1-x_{1}\right) \mu_{2}^{2}$ & 0 \\
\hline $2 \mathrm{a}$ & $\left(x_{1} \mu_{1}-I\right) \mu_{1}$ & $\left(1-x_{1}\right) \mu_{2}^{2}$ & 0 \\
\hline $2 \mathrm{~b}, \mathrm{~L}$ & $\left(x_{1} \mu_{1}+\left(1-x_{1}\right) \mu_{2}(1-c)-I\right) \mu_{1}$ & 0 & 0 \\
\hline $2 \mathrm{~b}, \mathrm{~K}$ & 0 & $\left(1-x_{1}\right) \mu_{2}^{2}$ & $\left(x_{1} \mu_{1}-I\right) \mu_{3}$ \\
\hline
\end{tabular}

Tabelle 2: Werte der einzelnen Assets in den unterschiedlichen Situationen

\subsection{Analyse der Zielfunktion}

Wie im vorhergehenden Abschnitt dargestellt, kann der Anleger - falls erforderlich - zwischen zwei Alternativen entscheiden. Entweder er liquidiert das illiquide Asset oder er wählt eine kurzfristige Fremdfinanzierung bis dieses verlustfrei liquidiert werden kann. Da im vorliegenden Beitrag die Auswirkungen der Kreditfinanzierung auf die optimale Allokation im Fokus stehen, wird im Folgenden keine integrierte Optimierung über beide Alternativen vorgenommen. Um feststellen zu können, welche der beiden Entscheidungen in welchen Situationen jeweils zum maximalen erwarteten Portfoliowert am Ende der zweiten Periode führt und wodurch sich diese unterscheiden, werden sie stattdessen zunächst getrennt analysiert und anschliessend gegenübergestellt:

\subsubsection{Der Anleger entscheidet sich für die Liquidation des illiquiden Assets}

Der erwartete Portfoliowert, falls der Anleger - wenn nötig - das illiquide Asset kurzfristig liquidiert $\left(E\left(V_{L}\left(x_{1}\right)\right)\right)$, setzt sich aus den Portfoliowerten $V_{(1)}, V_{(2 a)}$ und $V_{(2 b), L}$ jeweils gewichtet mit ihrer Eintrittswahrscheinlichkeit zusammen:

$$
\begin{aligned}
& E\left(V_{L}\left(x_{1}\right)\right)=\left(1-p_{I}\right) V_{(1)}\left(x_{1}\right)+p_{I}\left\{\begin{array}{c}
V_{(2 a)}\left(x_{1}\right), x_{1} \geq I / \mu_{1} \\
V_{(2 b), L}\left(x_{1}\right), x_{1}<I / \mu_{1}
\end{array}\right. \\
& =\left(1-p_{I}\right)\left(x_{1} \mu_{1}^{2}+\left(1-x_{1}\right) \mu_{2}^{2}\right)+p_{I}\left\{\begin{array}{c}
\left(\left(x_{1} \mu_{1}-I\right) \mu_{1}+\left(1-x_{1}\right) \mu_{2}^{2}\right), x_{1} \geq I / \mu_{1} \\
\left(x_{1} \mu_{1}+\left(1-x_{1}\right) \mu_{2}(1-c)-I\right) \mu_{1}, x_{1}<I / \mu_{1}
\end{array}, x_{1} \geq I / \mu_{1}\right. \\
& \Rightarrow E\left(V_{L}\left(x_{1}\right)\right)=x_{1} \mu_{1}^{2}-p_{I} I \mu_{1}+\left\{\begin{array}{c}
\left(1-x_{1}\right) \mu_{2}^{2} \\
\left(1-p_{I}\right)\left(1-x_{1}\right) \mu_{2}^{2}+p_{I}\left(1-x_{1}\right) \mu_{2}(1-c) \mu_{1}, x_{1}<I / \mu_{1}
\end{array}\right.
\end{aligned}
$$

Die erwartete Wertentwicklung des illiquiden Assets hängt demnach von der Liquiditätsanforderung ab. Der erwartete Portfoliowert ist stückweise linear.

Wie von Diepold/Dzienziol (2009) gezeigt wurde, kann hier nur entweder die vollständige Investition in eine der beiden Assetklassen oder das liquide Vorhalten des gesamten Liquiditätsbedarfs zur Maximierung des erwarteten Portfoliowerts führen. Durch die kon- 
stanten Liquidationskosten ergibt sich für den spannenden Bereich $\mu_{2}>\mu_{1}$ (s. u.) immer ein Sprung bei $x_{1}=I / \mu_{1}$, da das illiquide Asset ab diesem Anteil sicher nicht mehr liquidiert werden muss. ${ }^{5}$ Zusammen mit der stückweisen Linearität des erwarteten Portfoliowerts ergibt dies folgende drei mögliche Situationen: ${ }^{6}$

(LI) $\mu_{1} \geq \mu_{2}$ :

Die Rendite des liquiden Assets ist grösser gleich der Rendite des illiquiden Assets. Hier ist die vollständige Investition in das liquide Asset optimal, da dieses zudem kein Liquidationsrisiko beinhaltet.

(LII) $\mu_{2}>\mu_{1} \wedge E\left(V_{L}(0)\right) \leq E\left(V_{L}\left(I / \mu_{1}\right)\right)$

$\Leftrightarrow\left(1-p_{l}\right) \mu_{2}^{2}+p_{1} \mu_{2}(1-c) \mu_{1} \leq\left(I / \mu_{1}\right) \mu_{1}^{2}+\left(1-I / \mu_{1}\right) \mu_{2}^{2}:$

Die erwartete Zwei-Perioden-Wertentwicklung des illiquiden Assets ist kleiner gleich der sicheren Wertentwicklung des Portfolios bei vorgehaltenem Liquiditätsbedarf im liquiden Asset. In dieser Situation wird der diskontierte Liquiditätsbedarf im liquiden Asset angelegt, weil hier die durch die Anlage im renditeschwächeren liquiden Asset entgangene Rendite geringer als die dadurch verhinderten erwarteten Liquidationskosten ist (vgl. Abbildung 1).

(LIII) $E\left(V_{L}(0)\right)>E\left(V_{L}\left(I / \mu_{1}\right)\right)$ :

Die erwartete Zwei-Perioden-Wertentwicklung des illiquiden Assets ist grösser als die sichere Wertentwicklung des Portfolios bei vorgehaltenem Liquiditätsbedarf im liquiden Asset. Der Anleger investiert daher vollständig in das illiquide Asset (vgl. Abbildung 2).

\subsubsection{Der Anleger entscheidet sich für den Kredit}

Wählt der Anleger falls notwendig die Fremdfinanzierung des Liquiditätsbedarfs, so erhält man für den Portfoliowert am Ende der zweiten Periode:

$$
\begin{aligned}
& E\left(V_{K}\left(x_{1}\right)\right)=\left(1-p_{I}\right) V_{(1)}\left(x_{1}\right)+p_{I}\left\{\begin{array}{c}
V_{(2 a)}\left(x_{1}\right), x_{1} \geq I / \mu_{1} \\
V_{(2 b), K}\left(x_{1}\right), x_{1}<I / \mu_{1}
\end{array}\right. \\
& =\left(1-p_{I}\right)\left(x_{1} \mu_{1}^{2}+\left(1-x_{1}\right) \mu_{2}^{2}\right)+p_{I}\left\{\begin{array}{l}
\left(\left(x_{1} \mu_{1}-I\right) \mu_{1}+\left(1-x_{1}\right) \mu_{2}^{2}\right), x_{1} \geq I / \mu_{1} \\
\left(0+\left(1-x_{1}\right) \mu_{2}^{2}-\left(I-x_{1} \mu_{1}\right) \mu_{3}\right), x_{1}<I / \mu_{1}
\end{array}\right. \\
& \Rightarrow E\left(V_{K}\left(x_{1}\right)\right)=\left(1-p_{I}\right) x_{1} \mu_{1}^{2}+\left(1-x_{1}\right) \mu_{2}^{2}+p_{I}\left\{\begin{array}{l}
\left(x_{1} \mu_{1}-I\right) \mu_{1}, x_{1} \geq I / \mu_{1} \\
\left(x_{1} \mu_{1}-I\right) \mu_{3}, x_{1}<I / \mu_{1}
\end{array}\right.
\end{aligned}
$$

Der erwartete Portfoliowert ist demnach ebenfalls stückweise linear ${ }^{7}$ und die optimale Aufteilung hängt von der Liquiditätsanforderung ab. Für das optimale $x_{1}$ gilt somit auch hier $x_{1} \in\left\{0 ; I / \mu_{1} ; 1\right\}$, d. h. es ist für den Anleger nicht sinnvoll, nur einen Teil der Liquiditätsanforderung liquide vorzuhalten (falls er nicht indifferent zwischen den beiden Assets

5 Es gilt: $\lim _{\varepsilon \rightarrow 0} E\left(V_{L}\left(I / \mu_{1}-\varepsilon\right)\right)<E\left(V_{L}\left(I / \mu_{1}\right)\right)$, für $\varepsilon>0$ und $\mu_{2}>\mu_{1}(1-c)$.

6 Bzgl. Herleitung und Erläuterung der Zusammenhänge siehe Diepold/Dzienziol (2009).

$7 \frac{\partial E\left(V_{K}\left(x_{1}\right)\right)}{\partial x_{1}}=\left(1-p_{I}\right) \mu_{1}^{2}-\mu_{2}^{2}+p_{I}\left\{\begin{array}{cc}\mu_{1}^{2} \\ \mu_{1} \mu_{3}\end{array}=-\mu_{2}^{2}+\left\{\begin{array}{cc}\mu_{1}^{2} & , x_{1} \geq I / \mu_{1} \\ \left(1-p_{I}\right) \mu_{1}^{2}+p_{I} \mu_{1} \mu_{3} & , x_{1}<I / \mu_{1}\end{array}\right.\right.$. 
ist). Grund dafür ist, dass die Höhe der nötigen Finanzierung $\left(I-x_{1} \mu_{1}\right)$ kontinuierlich mit steigendem Anteil des liquiden Assets sinkt, bis der Wert des Liquiditätsbedarfs erreicht ist und bleibt dann konstant 0. Daraus folgt zudem, dass der erwartete Portfoliowert über $x_{1}=I / \mu_{1}$ stetig ist ${ }^{8}$ und dessen Steigung für $x_{1}<I / \mu_{1}$ stets grösser ist, als für $x_{1} \geq I / \mu_{1} \cdot{ }^{9}$

Im Folgenden soll in Abhängigkeit der Kreditkosten genau untersucht werden, wann welche der drei relevanten Aufteilungen $\left(x_{1} \in\left\{0 ; I / \mu_{1} ; 1\right\}\right)$ die Zielfunktion maximiert, falls eine Liquidation des illiquiden Assets nicht in Betracht gezogen wird. Die einzelnen Fälle lassen sich über die Steigung der Zielfunktion differenzieren.

Für $x_{1}<I / \mu_{1}$ erhält man hier:

$$
\begin{aligned}
& \frac{\partial E\left(V_{K}\left(x_{1}\right)\right)}{\partial x_{1}}=\left(1-p_{l}\right) \mu_{1}^{2}-\mu_{2}^{2}+p_{l} \mu_{1} \mu_{3} \stackrel{!}{=} 0 \Leftrightarrow\left(1-p_{l}\right) \mu_{1}^{2}+p_{l} \mu_{1} \mu_{3} \stackrel{!}{=} \mu_{2}^{2} \\
\Leftrightarrow & \mu_{3} \stackrel{!}{=} \frac{\mu_{2}^{2}-\left(1-p_{l}\right) \mu_{1}^{2}}{p_{l} \mu_{1}}=: S K
\end{aligned}
$$

Der erwartete Portfoliowert steigt demnach genau dann mit $x_{1}$ im Bereich $x_{1}<I / \mu_{1}$, wenn $\left(1-p_{I}\right) \mu_{1}^{2}+p_{I} \mu_{1} \mu_{3}>\mu_{2}^{2}$, das heisst wenn der erwartete Wertbeitrag des liquiden Assets grösser als die Wertentwicklung des illiquiden Assets ist $\left(\mu_{2}^{2}\right)$. Ersterer setzt sich zusammen aus der Zwei-Perioden-Wertentwicklung des liquiden Assets $\left(\mu_{1}^{2}\right)$, falls kein Liquiditätsbedarf eintritt, sowie der Wertentwicklung der ersten Periode und der Kreditkostenersparnis der zweiten Periode, welche durch die Investition in das liquide Asset entsteht $\left(\mu_{1} \mu_{3}\right)$, sonst. Für $\mu_{3}=S K$ ist der Anleger in diesem Bereich indifferent zwischen den beiden Assets.

Im Bereich von $x_{1} \geq I / \mu_{1}$ ist die Steigung unabhängig von den Kreditkosten, da hier keine Kreditaufnahme notwendig ist und der erwartete Portfoliowert mit dem erwarteten Portfoliowert bei Entscheidung für die Liquidation des illiquiden Assets übereinstimmt.

Kommt ausschliesslich die Aufnahme des Kredits in Betracht, so sind damit folgende drei Situationen zu unterscheiden:

(KI) $\left(\mu_{3}>\right) \mu_{1} \geq \mu_{2}$ : Die Wertentwicklung des liquiden Assets ist mindestens so gross wie die des illiquiden Assets, welche nach Voraussetzung beide kleiner als die Kostenentwicklung des Kredits sind. Wie in (LI) ist es auch hier optimal, den gesamten Anlagebetrag in das liquide Asset zu investieren.

(KII) $\mu_{3} \geq S K \geq \mu_{2}>\mu_{1}$ : Der Kredit ist so teuer, dass es sich lohnt in das liquide Asset zu investieren, obwohl dessen Rendite geringer als die des illiquiden Assets ist. Der Anteil des liquiden Assets wird so lange erhöht, bis sein Wert ausreicht, um die Liquiditätsanforderung abzudecken. Eine weitere Erhöhung dieses Anteils ist nicht sinnvoll, da sie zu keiner weiteren Reduzierung des Kreditbetrags führt und die Rendite des illiquiden Assets höher ist. In dieser Situation ist es also optimal, den eventuell benö-

$8 \lim _{\varepsilon \rightarrow 0} E\left(V_{K}\left(l / \mu_{1}-\varepsilon\right)\right)=\left(1-p_{I}\right) x_{1} \mu_{1}^{2}+\left(1-x_{1}\right) \mu_{2}^{2}=E\left(V_{K}\left(I / \mu_{1}\right)\right)$.

9 Mit $\mu_{3}>\mu_{1}$ folgt: $p_{I} \mu_{1} \mu_{3}>p_{I} \mu_{1} \mu_{1} \Leftrightarrow \frac{\left.\partial E\left(V_{K}\left(x_{1}\right)\right)\right|_{X_{1}<l / \mu_{1}}}{\partial x_{1}}>\frac{\left.\partial E\left(V_{K}\left(x_{1}\right)\right)\right|_{X_{1}} \geq l / \mu_{1}}{\partial x_{1}}$. 
tigten Betrag genau durch das liquide Asset bereitzustellen. Dies entspricht der gleichen Aufteilung wie in Situation (LII).

(KIII) $S K>\mu_{3} \geq \mu_{2}>\mu_{1}$ : Der Kredit ist so günstig, dass eine Investition in das liquide Asset nicht sinnvoll ist. Der durch das Bereitstellen des Liquiditätsbedarfs im liquiden Asset entgangene Gewinn wäre grösser als die Kosten für eine eventuell benötigte Kreditaufnahme. Damit wird - wie in Situation (LIII) - vollständig in das renditestärkere illiquide Asset investiert (vgl. Abbildungen 1 und 2).

\subsubsection{Vergleich der beiden Alternativen zur Liquiditätsbeschaffung}

Im Folgenden wird durch Vergleich der beiden untersuchten Zielfunktionen festgestellt, welche Entscheidung (Liquidation versus Kredit) jeweils zu wählen ist und welche Aufteilung der beiden Assets letztlich den erwarteten Portfoliowert maximiert.

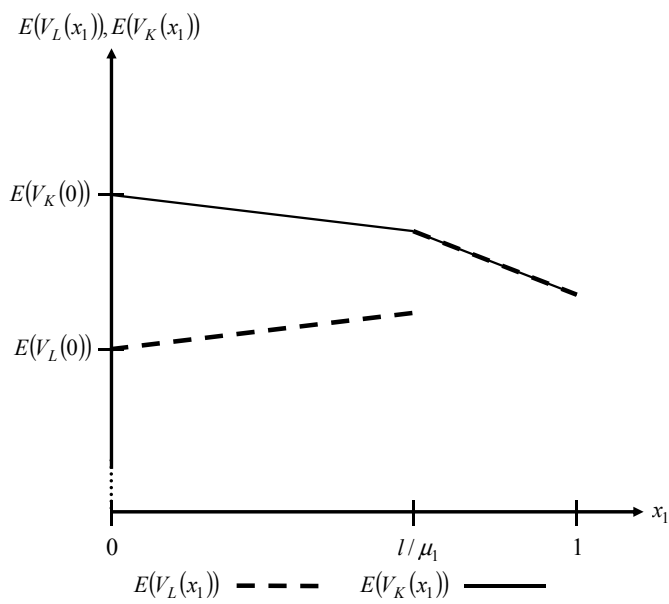

Abbildung 1: Beispiel unterschiedlicher Optima

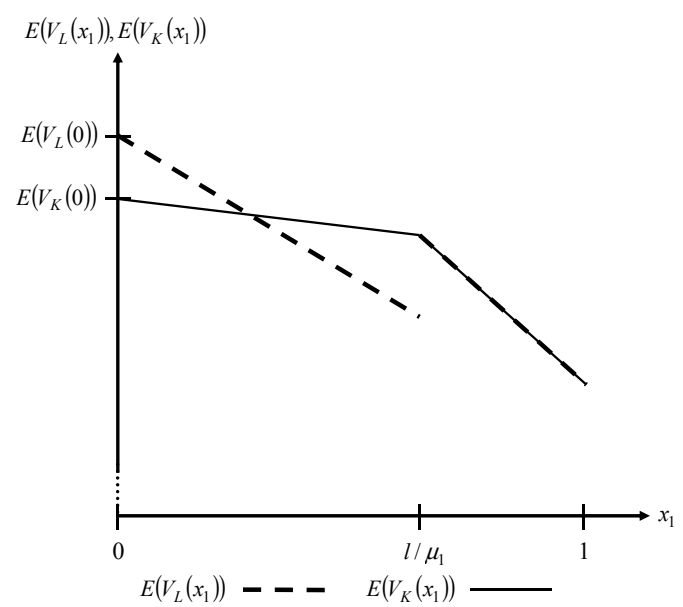

Abbildung 2: Beispiel eines gleichen Optimums

Abbildungen 1 und 2 zeigen zur Verdeutlichung dieses Vorgehens zwei beispielhafte Verläufe des erwarteten Portfoliowerts bei Liquidation des illiquiden Assets $\left(E\left(V_{L}\left(x_{1}\right)\right)\right) \mathrm{im}$ Vergleich zu einer Kreditaufnahme $\left(E\left(V_{K}\left(x_{1}\right)\right)\right)$, falls die Kreditkosten kleiner $S K$ sind. Hier sieht man nochmals, dass die beiden Zielfunktionen für $x_{1} \geq I / \mu_{1}$ identisch sind. Die kurzfristige Fremdfinanzierung ermöglicht in diesem Fall einen höheren maximalen erwarteten Portfoliowert. Derart niedrige Kreditkosten reduzieren die Nachteile des illiquiden Assets (unteilbarer Verkaufsverlust) so stark, dass immer vollständig in das illiquide Asset investiert wird, d. h. es ist nicht mehr sinnvoll, den Liquiditätsbedarf liquide vorzuhalten.

Sind die Kreditkosten grösser gleich SK (vgl. bspw. Abbildung 3), so hat die Möglichkeit zur Kreditaufnahme keinen Einfluss auf den maximalen Portfoliowert. Dabei ist zu beachten, dass eine Investition in das liquide Asset mit steigender Differenz zwischen der Rendite des liquiden und des illiquiden Assets immer weniger sinnvoll wird (das ist von Situation (LI) zu (LII) zu (LIII) tendenziell der Fall) und damit die Vorteilhaftigkeit des Kredits gegenüber einer Investition in das liquide Asset steigt. 
Zusammenfassend lässt sich folgendes Ergebnis festhalten:

Ergebnis 1:

Sieht sich ein Anleger bei der optimalen Aufteilung seines Anlagebetrags auf ein liquides und ein illiquides Asset mit jeweils sicherer Wertentwicklung einer möglichen Liquiditätsanforderung gegenüber, so ist der Portfoliowert dennoch unsicher. Das gilt auch dann, wenn der Anleger den Differenzbetrag zwischen vorhandener und benötigter Liquidität über einen Kredit bis zur verlustfrei möglichen Veräusserung des illiquiden Assets kurzfristig fremdfinanzieren kann. Im Unterschied zu einer Portfoliooptimierung mit liquiden Assets kann somit eine echte Aufteilung auf beide Assets optimal sein, nämlich genau die Investition des diskontierten Liquiditätsbedarfs in das liquide Asset. Dies ist genau dann der Fall, wenn die Wertentwicklung des liquiden Assets zuzüglich der erwarteten Kreditkostenersparnis durch die Investition in das liquide Asset grösser als die Wertentwicklung des illiquiden Assets ist. Mit anderen Worten: Ist der Kredit sehr teuer beziehungsweise die Differenz der Renditen des liquiden und illiquiden Assets gering, so ist es sinnvoll, den evtl. benötigten Liquiditätsbedarf im liquiden Asset vorzuhalten. Andernfalls erhöht die Möglichkeit der Kreditfinanzierung die Attraktivität des illiquiden Assets, insbesondere bei geringen Fehlbeträgen, da die Kreditkosten lediglich für den Differenzbetrag anfallen. Bei sehr günstigen Konditionen führt dies dazu, dass vollständig in das illiquide Asset investiert wird, auch wenn ohne diese Möglichkeit die Mischlösung gewählt werden würde. Dadurch wird der maximale erwartete Portfoliowert erhöht. Die Vernachlässigung der Möglichkeit der Kreditfinanzierung kann demnach zu Fehlentscheidungen bei der Portfolioselektion fübren.

Tabelle 3 zeigt für die unterschiedlichen Konstellationen jeweils den Anteil des liquiden Assets, der den erwarteten Portfoliowert maximiert sowie die jeweils bessere Alternative zur Deckung des Liquiditätsbedarfs:

\begin{tabular}{|l|c|l|c|c|}
\hline \multirow{2}{*}{$\begin{array}{l}\text { Situation } \\
\text { im }\end{array}$} & \multicolumn{2}{|c|}{$\mu_{3}<S K$} & \multicolumn{2}{c|}{$\mu_{3} \geq S K$} \\
\cline { 2 - 5 } & $\begin{array}{l}\text { Opt. Anteil d. } \\
\text { liqu. Asssets }\end{array}$ & $\begin{array}{l}\text { Liquiditäts- } \\
\text { deckung (durch) }\end{array}$ & $\begin{array}{l}\text { Opt. Anteil d. } \\
\text { liqu. Assets }\end{array}$ & $\begin{array}{l}\text { Liquiditäts- } \\
\text { deckung (durch) }\end{array}$ \\
\hline (LI) & \multicolumn{2}{|c|}{ nicht möglich10 } & 1 & nicht nötig \\
\hline (LII) & 0 & Kredit & $I / \mu_{1}$ & nicht nötig \\
\hline (LIII) & 0 & $\begin{array}{c}\text { Kredit oder Liqui- } \\
\text { dation }\end{array}$ & 0 & Liquidation \\
\hline
\end{tabular}

Tabelle 3: Optimale Entscheidungen und zugehörige Portfolioaufteilungen

10 In Situation (LI) gilt $\mu_{1} \geq \mu_{2}$. Damit folgt: $S K \leq \mu_{2}<\mu_{3}$.

$11 \mathrm{Ob}$ im Falle eines Liquiditätsbedarfs die Liquidation des illiquiden Assets oder die Kreditaufnahme besser ist, hängt davon ab, welche Entscheidung den höheren Portfoliowert hat (vgl. Abbildung 2). Es gilt:

$$
\begin{aligned}
& E\left(V_{K}(0)\right) \geq E\left(V_{L}(0)\right) \Leftrightarrow \mu_{2}^{2}-p_{I} I \mu_{3} \geq-p_{I} I \mu_{1}+\left(1-p_{I}\right) \mu_{2}^{2}+p_{I} \mu_{2}(1-c) \mu_{1} \\
& \Leftrightarrow \mu_{2}^{2}-I \mu_{3} \geq-I \mu_{1}+\mu_{2}(1-c) \mu_{1} \Leftrightarrow V_{(2 b), K^{(0)} \geq V_{(2 b), L}}(0) .
\end{aligned}
$$


Im Folgenden werden die bisherigen Ergebnisse an einem Beispiel illustriert:

Einem Anleger stehen ein Tagesgeldkonto mit 3\% Zins p.a. und eine Lebensversicherung mit einer jährlichen Rendite von $4 \%$ für die Investition eines gewissen Kapitals zur Verfügung. Bei einem Verkauf der Lebensversicherung vor Ablauf von 12 Jahren ergibt sich ein Verlust von 5\% des aktuellen Werts. Der Anleger rechnet mit einer Wahrscheinlichkeit von $80 \%$ allerdings nach 6 Jahren mit einem Liquiditätsbedarf von $50 \%$ des Anlagebetrags. Diesen Bedarf kann er auch mit einem Kredit mit 6\% effektivem Jahreszins so lange fremdfinanzieren, bis die Lebensversicherung nach weiteren 6 Jahren fällig wird. Für die Parameter des Modells ergibt sich mit diesen Angaben: $\mu_{1}=1,03^{6}, \mu_{2}=1,04^{6}, c=$ $0,05, p_{l}=0,8, l=0,5, \mu_{3}=1,06^{6}$.

Wie Abbildung 3 zeigt, erhält man in dieser Situation den maximalen erwarteten Portfoliowert, wenn man den eventuell benötigten Betrag durch das liquide Asset bereitstellt. Da der Kredit zu teuer ist, hat dieser keinen Einfluss auf das Optimum.

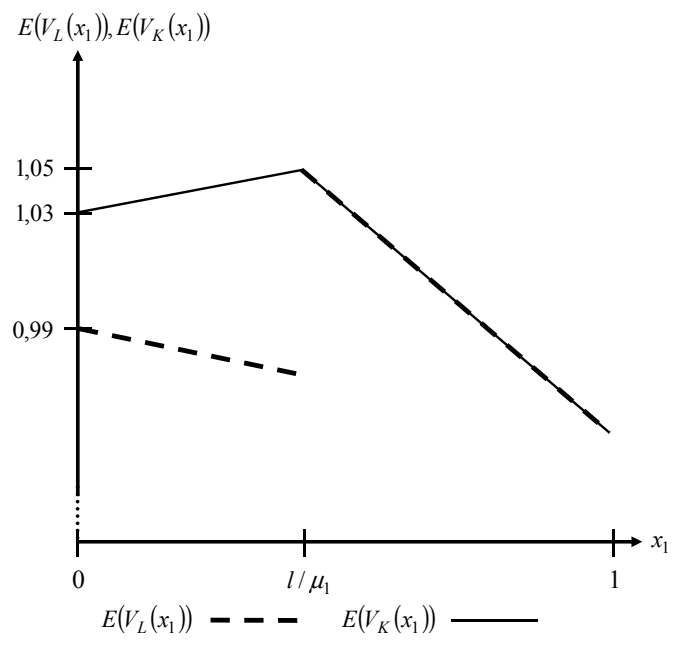

Abbildung 3: Erwarteter Portfoliowert im Beispiel

Dies ändert sich, sobald es einen Kredit gibt, dessen effektiver Jahreszins kleiner als 5,48\% $\left(\mathrm{SK}=1,3775=1,0548^{6}\right)$ ist. Dann wird das Maximum des erwarteten Portfoliowerts durch vollständige Investition in das illiquide Asset erreicht, da der Kredit so günstig ist, dass der erwartete entgangene Gewinn durch das Vorhalten im liquiden Asset grösser als die Kosten einer eventuell nötigen Kreditaufnahme ist.

\subsection{Analyse der Nebenbedingung}

Gemäss (A2) will der Anleger auch im Worst-Case ein gewisses Ziel ( $\omega$ ) nicht verfehlen, d. h. der Portfoliowert bei Eintreten der Liquiditätsanforderung $\left(V_{(2)}\right)$ muss mindestens den Wert $\omega$ erreichen. Für die beiden Arten der Liquiditätsbereitstellung ergeben sich dafür folgende Portfoliowerte: 


$$
\begin{aligned}
& V_{(2), L}\left(x_{1}\right)=\left\{\begin{array}{c}
V_{(2 a)}\left(x_{1}\right)=\left(x_{1} \mu_{1}-I\right) \mu_{1}+\left(1-x_{1}\right) \mu_{2}^{2} \quad, x_{1} \geq I / \mu_{1} \\
V_{(2 b), L}\left(x_{1}\right)=\left(x_{1} \mu_{1}+\left(1-x_{1}\right) \mu_{2}(1-c)-I\right) \mu_{1}, x_{1}<I / \mu_{1}
\end{array}\right. \\
& V_{(2), K}\left(x_{1}\right)=\left\{\begin{array}{l}
V_{(2 a)}\left(x_{1}\right)=\left(x_{1} \mu_{1}-I\right) \mu_{1}+\left(1-x_{1}\right) \mu_{2}^{2}, x_{1} \geq I / \mu_{1} \\
V_{(2 b), K}\left(x_{1}\right)=\left(x_{1} \mu_{1}-I\right) \mu_{3}+\left(1-x_{1}\right) \mu_{2}^{2}, x_{1}<I / \mu_{1}
\end{array}\right.
\end{aligned}
$$

Zunächst lässt sich analog zu (LI) und (KI) festhalten, dass $V_{(2), L}$ und $V_{(2), K}$ für $\mu_{1} \geq \mu_{2}$ ebenfalls ihr Maximum bei $x_{1}=1$ haben, weswegen die Nebenbedingung in diesem Fall keine Auswirkungen auf das Optimum haben kann.

Betrachtet man nun für $\mu_{1}<\mu_{2}$ die Differenz zwischen dem erwarteten Portfoliowert und $V_{(2), L}$ bzw. $V_{(2), K}$, so erhält man für $x_{1} \geq I / \mu_{1}$ :

$$
E\left(V\left(x_{1}\right)\right)-V_{(2 a)}\left(x_{1}\right)=\left(1-p_{I}\right) I \mu_{1}
$$

$V_{(2)}$ ist also in diesem Bereich konstant kleiner als der erwartete Portfoliowert und hat demnach dieselbe Steigung $\left(\mu_{1}^{2}-\mu_{2}^{2}\right) . V_{(2)}$ steigt in diesem Bereich somit mit steigendem $x_{1}$, falls $\mu_{1}>\mu_{2}$ und vice versa. Dies liegt daran, dass der einzige Unterschied in der zugrunde liegenden Wahrscheinlichkeit für das Eintreten der Liquiditätsanforderung liegt.

Für $x_{1}<I / \mu_{1}$ gilt $\left(\right.$ mit $\left.\mu_{1}<\mu_{2}\right)$ :

$$
E\left(V_{L}\left(x_{1}\right)\right)-V_{(2 b), L}\left(x_{1}\right)=\left(1-p_{I}\right) I \mu_{1}+\left(1-p_{I}\right)\left(1-x_{1}\right) \mu_{2}^{2}-\left(1-p_{I}\right)\left(1-x_{1}\right) \mu_{2}(1-c) \mu_{1}>\left(1-p_{I}\right) I \mu_{1}
$$

sowie

$$
E\left(V_{K}\left(x_{1}\right)\right)-V_{(2 b), K}\left(x_{1}\right)=\left(1-p_{I}\right) x_{1} \mu_{1}^{2}-\left(1-p_{I}\right)\left(x_{1} \mu_{1}-I\right) \mu_{3}>\left(1-p_{I}\right) I \mu_{1} \cdot{ }^{12}
$$

In diesem Bereich ist die Differenz zwischen dem erwarteten Portfoliowert und dem Portfoliowert bei Eintreten der Liquiditätsanforderung demnach immer grösser als für $x_{1} \geq I / \mu_{1}$. Grund dafür ist der zusätzliche Nachteil durch Liquidation bzw. Kreditaufnahme, der mit zunehmender Finanzierungslücke - d. h. sinkendem Anteil des liquiden Assets - steigt. Daraus folgt unmittelbar, dass die Nebenbedingung nur dann Auswirkungen auf die optimale Aufteilung haben kann, wenn der erwartete Portfoliowert bei $x_{1}=0$ erreicht wird, d. h. wenn der Anleger vollständig in das illiquide Asset investiert. Liegt das Maximum des erwarteten Portfoliowerts bei $x_{1}=I / \mu_{1}$ bzw. $x_{1}=1$, so muss auch $V_{(2)}$ an derselben Stelle sein Maximum aufweisen und kann damit nicht zu einer Veränderung des Optimums führen. Daher sind im Folgenden nur diejenigen Situationen zu untersuchen, in denen die vollständige Investition in das illiquide Asset den erwarteten Portfoliowert maximiert.

12 Für $x_{1}=0$ folgt: $\left(1-p_{I}\right) x_{1} \mu_{1}^{2}-\left(1-p_{l}\right)\left(x_{1} \mu_{1}-I\right) \mu_{3}=\left(1-p_{I}\right) I \mu_{3}>\left(1-p_{I}\right) I \mu_{1}$. Zudem hat $V_{(2)}$ für $x_{1}<I / \mu_{1}$ die konstante Steigung $\mu_{1} \mu_{3}-\mu_{2}^{2}$. Damit sinkt die Differenz zwischen erwartetem Portfoliowert und $V_{(2)}$ mit steigendem $x_{1}$ von $\left(1-p_{l}\right) I \mu_{3}$ auf $\left(1-p_{I}\right)^{l} \mu_{1}$. 


\subsubsection{Der Anleger entscheidet sich für die Liquidation des illiquiden Assets}

Betrachtet man die Entscheidung für die Liquidation des illiquiden Assets, so genügt es, Fall (LIII) zu analysieren. Hier wird vollständig in das illiquide Asset investiert, der erwartete Portfoliowert ist bis auf einen Sprung bei $x_{1}=I / \mu_{1}$ fallend in $x_{1}$.

Über die erste Ableitung des liquidierten Portfoliowerts

$$
\frac{\partial V_{(2 b), L}\left(x_{1}\right)}{\partial x_{1}}=\mu_{1}^{2}-\left\{\begin{array}{cc}
\mu_{2}^{2} & , x_{1} \geq I / \mu_{1} \\
\mu_{2}(1-c) \mu_{1}, & x_{1}<I / \mu_{1}
\end{array}\right.
$$

stellt man fest, dass dieser für $x_{1}<1 / \mu_{1}$ genau dann mit $x_{1}$ steigt, wenn $\mu_{1}>\mu_{2}(1-c)$. Es sind damit - wie im Modell von Diepold/Dzienziol (2009) - die folgenden beiden Situationen zu unterscheiden:

1. Situation $\mu_{1}>\mu_{2}(1-c)$ (vgl. Abbildung 4). Je nach Höhe der vom Anleger festgelegten Untergrenze $\omega$ gibt es folgende Möglichkeiten:

Ist die $\omega$ grösser als $V_{(2), L}(0)=\left(\mu_{2}(1-c)-I\right) \mu_{1}$, so ist die Nebenbedingung bindend für die Optimierung. Das Optimum verschiebt sich zunächst kontinuierlich mit Erhöhung von $\omega$ nach rechts: Um die Risikonebenbedingung zu erfüllen, muss demnach der Anteil des liquiden Assets erhöht werden. Diese kontinuierliche Verschiebung erfolgt allerdings nur bis zu demjenigen Anteil a, bei welchem der zugehörige erwartete Portfoliowert dem Wert in $x_{1}=I / \mu_{1}$ entspricht. Ab hier $\left(\omega>V_{(2), L}(a)\right)$ ist nun $x_{1}=I / \mu_{1}$ für alle noch erfüllbaren $\omega$ optimal, da für diese Aufteilung sowohl der erwartete Portfoliowert als auch $V_{(2)}$ maximal werden.

2. Situation $\mu_{1} \leq \mu_{2}(1-c)($ vgl. Abbildung 5): Die vollständige Investition in das illiquide Asset bleibt solange optimal, bis $\omega$ grösser als $V_{(2), L}(0)$ ist. Ist das der Fall, so springt das Optimum - falls die Nebenbedingung noch erfüllbar ist - wieder auf $x_{1}=I / \mu_{1}$.

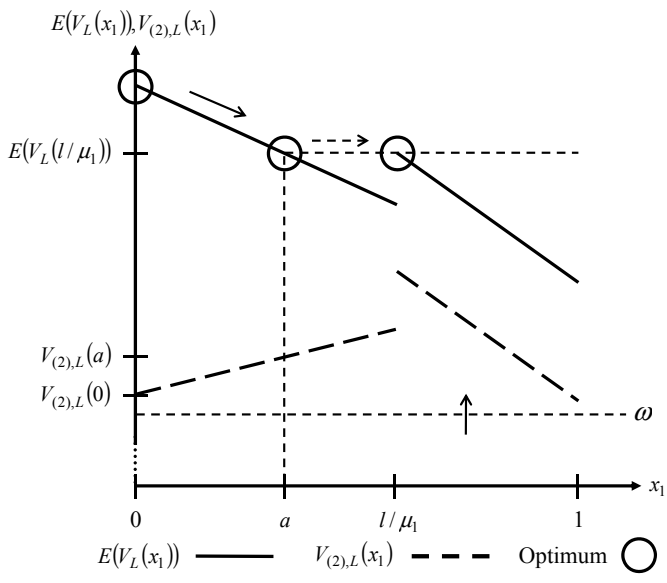

Abbildung 4: Einfluss der Nebenbedingung (1)

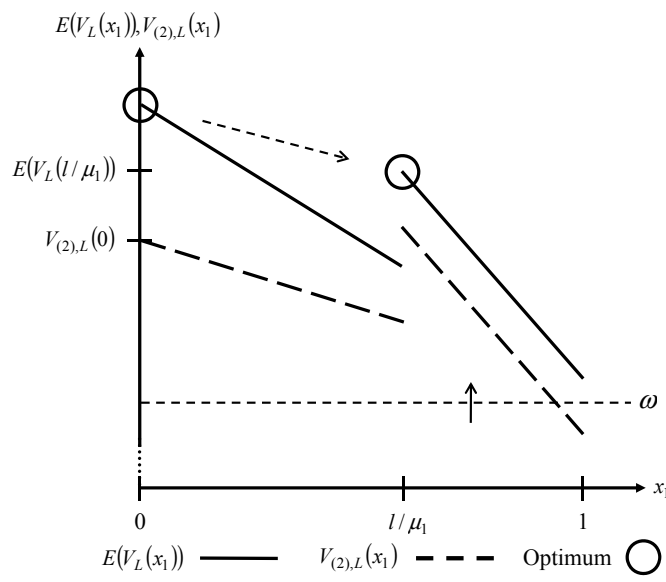

Abbildung 5: Einfluss der Nebenbedingung (2) 


\subsubsection{Der Anleger entscheidet sich für den Kredit}

Betrachtet man nur die Entscheidung für die Kreditaufnahme, so kann die optimale Aufteilung - da es keinen Sprung bei $x_{1}=I / \mu_{1}$ gibt - nur dann von einem Maximum der Zielfunktion bei $x_{1}=0$ abweichen, wenn $V_{(2), L}\left(x_{1}\right)$ mit $x_{1}$ steigt. Dies ist wegen

$$
\frac{\partial V_{(2), K}\left(x_{1}\right)}{\partial x_{1}}=\left\{\begin{array}{l}
\mu_{1}^{2}-\mu_{2}^{2}, x_{1} \geq I / \mu_{1} ! \\
\mu_{1} \mu_{3}-\mu_{2}^{2}, x_{1}<I / \mu_{1}>0
\end{array}\right.
$$

für $\mu_{3}>\mu_{2}^{2} / \mu_{1}$ der Fall. Mit steigendem $\omega$ wird der Anteil des liquiden Assets solange kontinuierlich erhöht, bis der gesamte Bedarf durch das liquide Asset abgedeckt ist (vgl. bspw. Abbildung 6).

\subsubsection{Vergleich der beiden Alternativen zur Liquiditätsbeschaffung}

Zur konkreten Bestimmung der optimalen Finanzierungsalternative und der zugehörigen Aufteilung der Assets unter Berücksichtigung der Nebenbedingung wird nun diejenige Alternative mit dem höheren Optimum gewählt. Ob und wie dabei letztlich die Nebenbedingung zu einer anderen optimalen Aufteilung (als der mit dem maximalen erwarteten Portfoliowert) und gegebenenfalls sogar zur Wahl einer anderen Finanzierungsalternative führen kann, wird im Folgenden untersucht.

Ergibt sich der maximale erwartete Portfoliowert durch Liquidation des illiquiden Assets, so sind folgende Möglichkeiten zu unterscheiden:

a) $\mu_{3} \geq S K$ : Hier ist die Möglichkeit der Kreditaufnahme irrelevant, da deren erwarteter Portfoliowert stets kleiner gleich dem Portfoliowert in $x_{1}=I / \mu_{1}$ und insbesondere dem darüber liegenden Teil des erwarteten Portfoliowerts unter Liquidation ist (vgl. Abbildung 3). Damit gelten die für die reine Liquidationsalternative dargestellten Ergebnisse. b) $\mu_{3}<S K$ (vgl. Abbildung 6): Ist der Kredit so günstig, dass eine Kreditfinanzierung der Anlage im liquiden Asset zwar vorgezogen, aber dennoch für höhere Finanzierungsbeträge durch die Liquidation des illiquiden Assets dominiert wird, so gilt für $x_{1}<I / \mu_{1}$ : $E\left(V_{K}\left(x_{1}\right)\right)>E\left(V_{L}\left(x_{1}\right)\right) \Leftrightarrow V_{(2), K}\left(x_{1}\right)>V_{(2), L}\left(x_{1}\right) \Leftrightarrow x_{1}>\frac{\mu_{2}^{2}-\mu_{1} \mu_{2}(1-c)+I\left(\mu_{1}-\mu_{3}\right)}{\mu_{2}^{2}-\mu_{1} \mu_{2}(1-c)+\mu_{1}\left(\mu_{1}-\mu_{3}\right)}=: b$

D. h. sowohl Zielfunktion als auch Nebenbedingung schneiden sich bei $x_{1}=b$. Grund dafür ist, dass mit steigendem Anteil des liquiden Assets immer weniger durch den Kredit fremdfinanziert werden muss und somit der Nachteil der ausschliesslich vollständigen Liquidationsmöglichkeit letztlich überwiegt. 


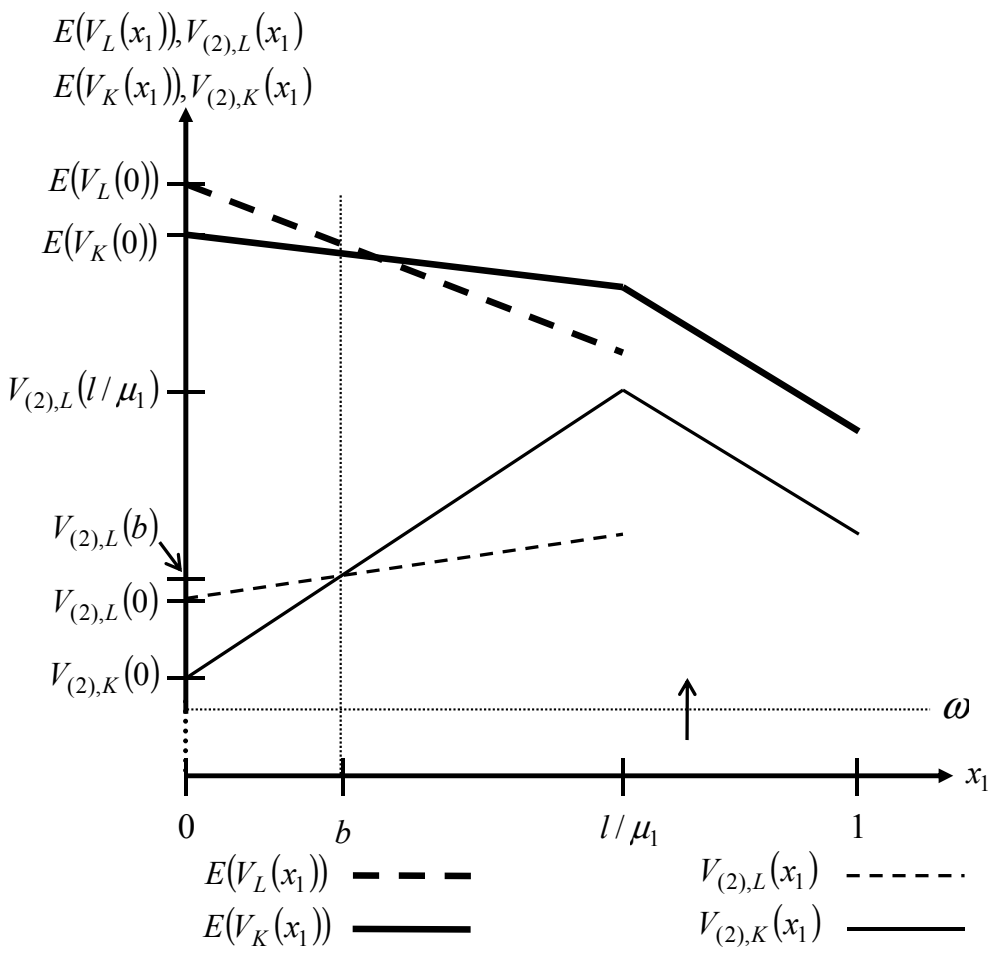

Abbildung 6: Zielfunktion und Nebenbedingung für Maximum $E\left(V_{L}(0)\right)$ und $\mu_{3}<S K$

Daraus folgt:

- Für $\omega \leq V_{(2), L}(0)$ ist die Nebenbedingung irrelevant,

- für $V_{(2), L}(0)<\omega \leq V_{(2), L}(b)=V_{(2), K}(b)$ erhöht sich das optimale $x_{1}$ bei Entscheidung für die Liquidation,

- für $V_{(2), K}(b)<\omega \leq V_{(2), K}\left(I / \mu_{1}\right)$ erhöht sich das optimale $x_{1}$ bei Entscheidung für den Kredit,

- für $V_{(2), K}\left(I / \mu_{1}\right)=V_{(2), L}\left(I / \mu_{1}\right)<\omega$ ist die Nebenbedingung nicht mehr erfüllbar.

Die alternative Kreditfinanzierung führt demnach im Bereich $x_{1} \in\left(b ; I / \mu_{1}\right]$ sowohl zu einer Verminderung des Risikos als auch zu einer Erhöhung der Rendite im Vergleich zur reinen Liquidationsmöglichkeit. Die Untergrenze $\omega$ kann sowohl zu einer Erhöhung des Anteils des liquiden Assets als auch einem Wechsel in der Vorteilhaftigkeit der Finanzierungsalternative führen.

Ergibt sich der maximale erwartete Portfoliowert durch die Möglichkeit der Kreditaufnahme, so ist dieser stets grösser gleich dem erwarteten Portfoliowert unter Liquidation. Dies gilt dann auch für den Portfoliowert bei Eintreten der Liquiditätsanforderung (vgl. Fussnote 11 sowie Abbildung 7). Für die optimale Aufteilung ist somit nur die Entscheidung für den Kredit relevant und die obigen Ergebnisse bleiben unverändert. 


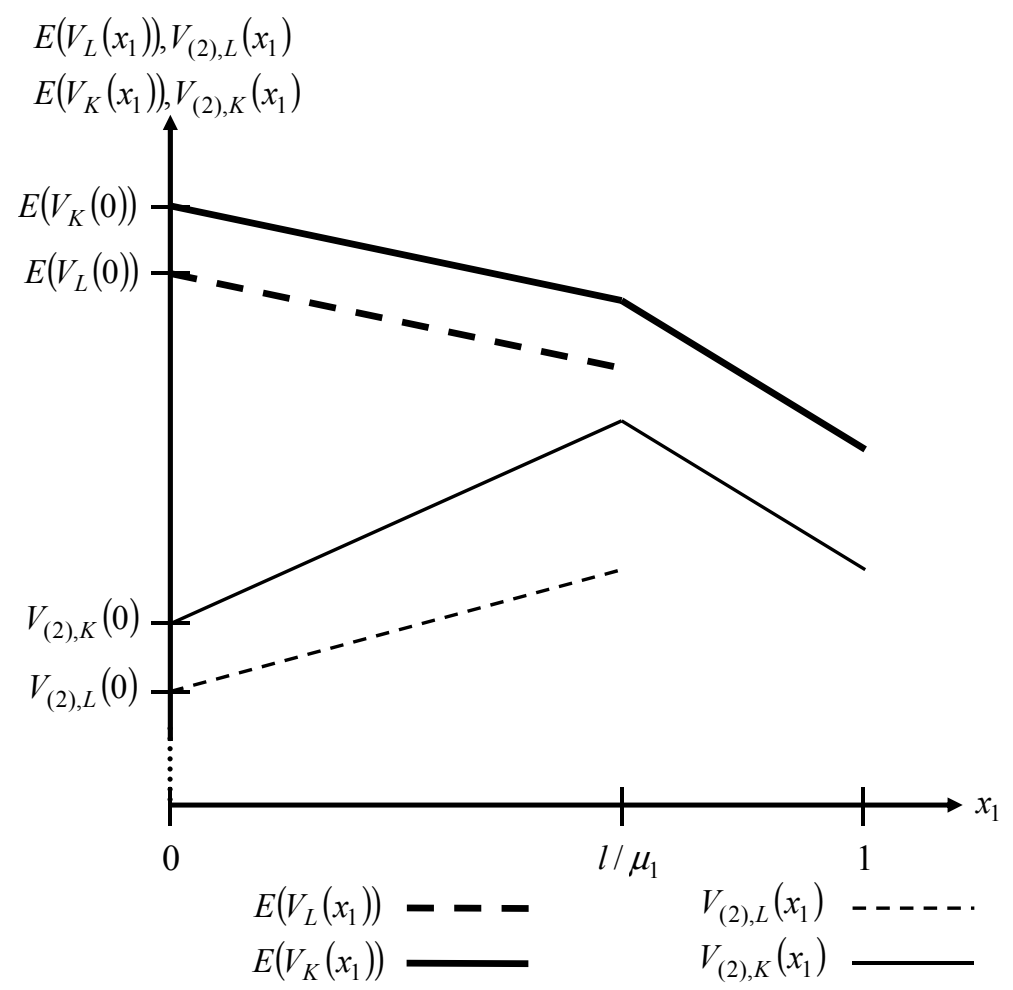

Abbildung 7: Zielfunktion und Nebenbedingung für Maximum $E\left(V_{K}(0)\right)$

Abschliessend kann somit folgendes Ergebnis festgehalten werden:

Ergebnis 2:

Durch die Berücksichtigung der Besonderheiten illiquider Assets ist die Nebenbedingung - eine Mindestanforderung an den Portfoliowert - trotz sicherer Renditen bereits relevant für das Optimierungsproblem und kann - im Gegensatz zur Optimierung mit liquiden Assets - Auswirkungen auf die optimale Portfoliostruktur haben. Dies kann auch bei einer Fremdfinanzierungsmöglichkeit nur dann der Fall sein, wenn die vollständige Investition in das illiquide Asset den erwarteten Portfoliowert maximiert. Das heisst, ist diese Lösung für den Anleger zu riskant, so wird durch die Nebenbedingung ein entsprechender Mindestanteil des liquiden Assets gefordert. Die Möglichkeit zur Kreditaufnahme beeinflusst die bisherigen Ergebnisse genau dann, wenn $\mu_{3}<S K$, wenn also der Kredit verhältnismässig billig ist. Ist dies der Fall, so wird der erwartete Portfoliowert durch die Möglichkeit der Kreditaufnahme abgesichert. Während der Anleger obne diese Möglichkeit ab einem gewissen Sicherheitslevel bereits den vollständigen Liquiditätsbedarf liquide vorhalten würde, kann er mit der Kreditalternative einen höheren Anteil im illiquiden Asset mit höherer Portfoliorendite und gleichzeitig niedrigerem Risiko realisieren. Die Nebenbedingung kann zudem einen Wechsel der optimalen Finanzierungsalternative bewirken. 


\section{Diskussion der Ergebnisse und weiterer Forschungsbedarf}

Das vorgestellte Modell erweitert die bisherige Literatur zur Portfoliooptimierung mit illiquiden Assets um die Berücksichtigung einer Fremdfinanzierungsalternative. Wenngleich dieses - wie auch die bisherige Literatur - weiterhin sehr spezifisch ist, geht es damit einen ersten Schritt in Richtung einer erhöhten Anwendbarkeit. Insbesondere liefert der vorliegende Beitrag unter den dargestellten Einschränkungen (ein liquides und ein illiquides Asset, Sicherheit bzgl. der Renditen sowie des Zeitpunkts und der Höhe der Liquiditätsanforderung) erste konkrete Ergebnisse sowie allgemeingültige Erkenntnisse bzgl. der Auswirkungen einer kurzfristigen Fremdfinanzierungsmöglichkeit. Er zeigt auf, dass diese bei der Anlage in illiquide Assets unbedingt zu berücksichtigen ist, und in welchen Fällen sich dadurch das optimale Portfolio verändert. Um die Anwendbarkeit des vorgestellten Modells weiter zu erhöhen besteht jedoch künftiger Forschungsbedarf.

Einen zentralen Punkt stellt dabei die Berücksichtigung unsicherer Renditen dar. Die Auswirkungen von Unsicherheit in den Renditen der Assets lassen sich in Verbindung mit den Ergebnissen von Diepold/Dzienziol (2009) wie folgt skizzieren: Bei unsicheren Renditen kann die Notwendigkeit zur Liquidation bzw. Fremdfinanzierung nicht mehr mit Sicherheit durch Anlage eines gewissen Anteils im liquiden Asset ausgeschlossen werden, weswegen der Portfoliowert über $I / \mu_{1}$ hinweg entsprechend der Verteilung der Renditen geglättet wird (d. h. es gibt keinen Sprung bzw. Knick mehr). Dadurch wird die Fremdfinanzierungsalternative in diesem Bereich im Vergleich zur Liquidation vorteilhafter, da nur der Differenzbetrag finanziert werden muss, falls die vorhandene Liquidität doch nicht ausreicht. Die Auswirkungen der Illiquidität werden damit auch im unsicheren Fall deutlich reduziert, was zu einer Erhöhung des Anteils des illiquiden Assets an einem inneren Optimum sowie des maximalen erwarteten Portfoliowerts führen kann.

Eine weitere Einschränkung besteht in der Fixierung des Zeitpunkts sowie der Höhe des Liquiditätsbedarfs. Da diese in der Realität oft ebenfalls unbekannt sind, könnte durch Berücksichtigung der entsprechenden Unsicherheiten die Anwendbarkeit weiter erhöht werden.

Darüber hinaus wäre es interessant, bestehende Modelle um die Berücksichtigung von steuerlichen Effekten (wie bspw. die durch die Abgeltungssteuer entstehende Steuerspreizung zwischen Kapitalanlagen und Immobilien) oder Informationsasymmetrien (bspw. bzgl. des Preisabschlags für den Verkauf eines illiquiden Assets) zu erweitern, da diese in der bisherigen Literatur zur Portfoliooptimierung mit illiquiden Assets nicht berücksichtigt werden.

\section{Zusammenfassung}

In der vorliegenden Arbeit wurde anhand eines zweiperiodigen Modells mit einem liquiden und einem illiquiden Asset mit jeweils sicherer Wertentwicklung der Einfluss einer Fremdfinanzierungsmöglichkeit auf die Portfoliooptimierung mit illiquiden Assets bei gegebenenfalls eintretender Liquiditätsanforderung untersucht. Die Besonderheiten illiquider Assets wurden dadurch definiert, dass das illiquide Asset stets nur vollständig verkauft werden kann und dessen Verkauf, wenn er kurzfristig erfolgt, Transaktionskosten verursacht. Alternativ zum kurzfristigen Verkauf des illiquiden Assets kann zur Bereitstellung ausreichender Liquidität ebenfalls ein Kredit aufgenommen werden. 
Es wurde gezeigt, dass durch die kurzfristige Fremdfinanzierungsmöglichkeit die Nachteile illiquider Assets tendenziell reduziert werden, ihre Besonderheiten jedoch weiterhin zu berücksichtigen sind. Sie führen dazu, dass auch bei sicheren Renditen Mischlösungen optimal sein können und eine Risikonebenbedingung - im Gegensatz zu einer Optimierung ohne Berücksichtigung von Illiquidität - einen Einfluss auf das Optimum haben kann. Die Möglichkeit der Kreditfinanzierung erhöht in bestimmten Situationen den Anteil des illiquiden Assets und den damit realisierbaren erwarteten Portfoliowert bei gleichzeitiger Reduzierung des Risikos, da sie zur Absicherung des Portfolios beiträgt. Grund dafür ist, dass das illiquide Asset nicht vollständig verkauft werden muss, sondern es ausreicht, lediglich die Differenz zwischen liquidem Asset und Liquiditätsbedarf zu finanzieren. Greift die Nebenbedingung nicht (oder entscheidet der Anleger nur auf Basis des erwarteten Portfoliowerts), so kann es auch bei einer möglichen Kreditaufnahme nur optimal sein, entweder vollständig in eines der beiden Assets zu investieren oder den gesamten Liquiditätsbedarf durch Investition in das liquide Asset bereitzustellen.

Der vorliegende Beitrag liefert somit erste Ergebnisse bezüglich der Auswirkungen einer kurzfristigen Fremdfinanzierungsmöglichkeit im Rahmen einer Portfoliooptimierung mit illiquiden Assets. Er zeigt auf, dass diese unbedingt Berücksichtigung finden muss, um Fehlentscheidungen zu vermeiden und gibt Anregungen für weitere Forschungsarbeiten.

\section{Literaturhinweise}

Bernstein, P.L. (1987): Liquidity, Stock Markets, and Market Makers, in: Financial Management, Jg. 16, Nr. 2, S. 54-62.

Diamond, D.W./Dybvig, P.H. (1983): Bank Runs, Deposit Insurance, and Liquidity, in: The Journal of Political Economy, Jg. 91, Nr. 3, S. 401-419.

Diepold, D./Dzienziol, J. (2009): Illiquide Assets in der Portfoliooptimierung, in: Zeitschrift für Betriebswirtschaft, Jg. 79, Nr. 10, S. 1143-1173.

Diepold, D. (2011): Zur Berücksichtigung illiquider Anlagen bei der privaten Finanzplanung, in: Wirtschaftswissenschaftliches Studium, Jg. 40, Nr. 11, S. 564-570.

Elton, E.J./Gruber, M.J. (2000): The Rationality of Asset Allocation Recommendations, in: The Journal of Financial and Quantitative Analysis, Jg. 35, Nr. 1, S. 27-41.

Ericsson, J./Renault, O. (2006): Liquidity and Credit Risk, in: The Journal of Finance, Jg. 61, Nr. 5, S. 2219-2250.

Faig, M./Shum, P. (2002): Portfolio Choice in the Presence of Personal Illiquid Projects, in: The Journal of Finance, Jg. 57, Nr. 1, S. 303-328.

Flavin, M./Yamashita, T. (2002): Owner-Occupied Housing and the Composition of the Household Portfolio, in: The American Economic Review, Jg. 92, Nr. 1, S. 345-362.

Gârleanu, N. (2009): Portfolio Choice and Pricing in Illiquid Markets, in: The Journal of Economic Theory, Jg. 144, Nr. 2, S. 532-564.

Grossmann, S.J./Laroque, G. (1990): Asset Pricing and Optimal Portfolio Choice in the Presence of Illiquid Durable Consumption Goods, in: Econometrica, Jg. 58, Nr. 1, S. 25-51.

Grossmann, S.J./Miller, M.H. (1988): Liquidity and Market Structure, in: The Journal of Finance, Jg. 43, Nr. 3, S. 617-633.

Heflin, F./Shaw, K.W. (2000): Blockholder Ownership and Market Liquidity, in: The Journal of Financial and Quantitative Analysis, Jg. 35, Nr. 4, S. 621-633. 
Huang, M. (2003): Liquidity Shocks and Equilibrium Liquidity Premia, in: The Journal of Economic Theory, Jg. 109, S. 104-129.

Jankowitsch, R./Nashikkar, A./Subrahmanyam, M.G. (2011): Price Dispersion in OTC Markets: A New Measure of Liquidity, in: The Journal of Banking \& Finance, Jg. 35, Nr. 2, S. 343-357.

Kempf, A. (1998): Was messen Liquiditätsmasse?, in: Die Betriebswirtschaft, Jg. 58, Nr. 3, S. 299-311.

Keynes, J.M. (1932): Vom Gelde, München.

Koren, M./Szeidl, Á. (2002): Portfolio Choice with Illiquid Assets, Working Paper, Harvard University, http://195.113.12.58/pdf/gdn/RRCI_28_paper_01.pdf, Abruf am 17.9.2010.

Lippman, S.A./McCall, J.J. (1986): An Operational Measure of Liquidity, in: The American Economic Review, Jg. 76, Nr. 1, S. 43-55.

Longstaff, F.A. (2009): Portfolio Claustrophobia: Asset Pricing in Markets with Illiquid Assets, in: The American Economic Review, Jg. 99, Nr. 4, S. 1119-1144.

Rudolf, M. (2008): Mehr als Markowitz, in: Die Bank, Jg. 2008, Nr. 4, S. 40-42.

Schwartz, E./Tebaldi, C. (2008): Illiquid Assets and Optimal Portfolio Choice, Working Paper, Cambridge, http://workshopfinance.ie.edu/files/Eduardo_Schwartz.pdf, Abruf am 17.9.2010.

Sibilkov, V. (2009): Asset Liquidity and Capital Structure, in: The Journal of Financial and Quantitative Analysis, Jg. 44, Nr. 5, S. 1173-1196.

Spremann, K./Winhart, S. (1997): Humankapital im Portefeuille privater Investoren, in: Zeitschrift für Betriebswirtschaft, Jg. 67, Nr. 1, S. 145-167.

Telser, L.G. (1955): Safety First and Hedging, in: The Review of Economic Studies, Jg. 23, S. 1-16.

Dennis Diepold, Dr., ist wissenschaftlicher Mitarbeiter am Lehrstuhl für BWL, Wirtschaftsinformatik, Informations- und Finanzmanagement und am Kernkompetenzzentrum Finanz- \& Informationsmanagement der Universität Augsburg.

Anschrift: FIM Kernkompetenzzentrum, Universität Augsburg, D-86135 Augsburg, Tel.: +49 (0)821/598-4862, Fax: +49 (0)821/598-4899, E-Mail: dennis.diepold@wiwi.uni-augsburg.de, URL: www.fim-online.eu 